\title{
Actividad ovárica y prolificidad de cabras sincronizadas con progestágenos y suplementadas con propionato de calcio
}

\author{
Ovarian activity and prolificity of goats synchronized with progestages and \\ supplemented with calcium propionate
}

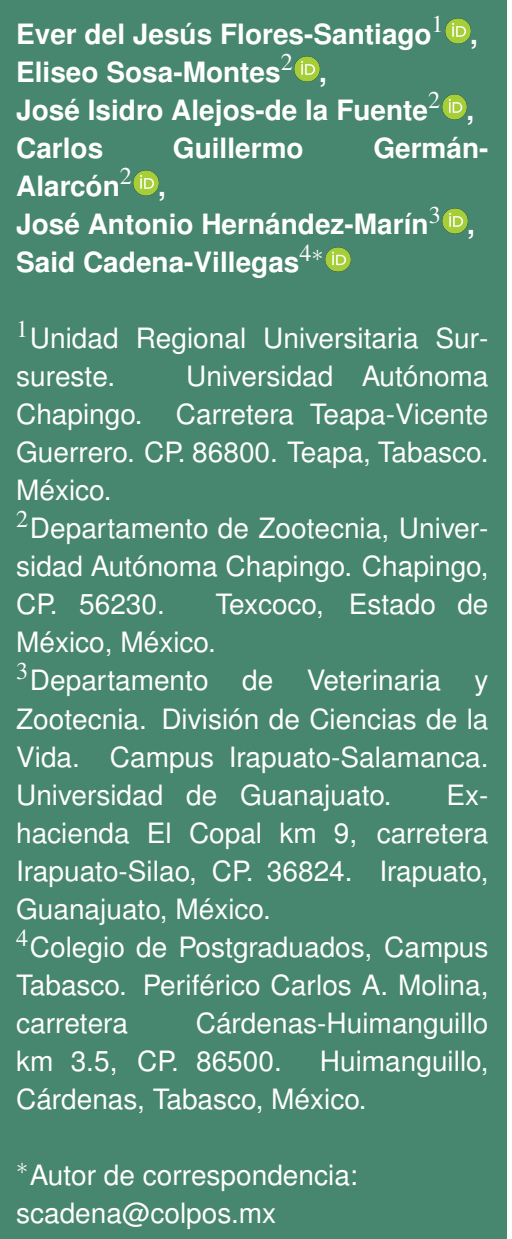

Artículo científico

Recibido: 08 de marzo 2021 Aceptado: 03 de septiembre 2021

Como citar: Flores-Santiago EJ, Sosa-Montes E, Alejos-de la Fuente JI, Germán-Alarcón CG, HernándezMarín JA, Cadena-Villegas S (2021) Actividad ovárica y prolificidad de cabras sincronizadas con progestágenos y suplementadas con propionato de calcio. Ecosistemas y Recursos Agropecuarios Núm. Esp. II: e2975. DOI: 10.19136/era.a8nll.2975
RESUMEN. Se sabe que el peso vivo (PV) y la ingesta energética inciden en la fertilidad de los caprinos, así como los beneficios de suministrar propionato de calcio (PC) como precursor de glucosa en sangre. El objetivo fue determinar la respuesta fisiológica y reproductiva de cabras sincronizadas en estro y un suplemento con PC. Los tratamientos se asignaron aleatoriamente a 25 cabras de $36.2 \pm 8.7$ meses y $51.36 \pm 0.47 \mathrm{~kg}$ de PV: T1 Sin propionato $(\mathrm{n}=13) 60 \mathrm{~mL}$ de agua sin PC, y T2 Con propionato $(\mathrm{n}=12) 20 \mathrm{~g}$ de $\mathrm{PC}$ diluido en $60 \mathrm{~mL}$ de agua. EI PC se administró vía oral diariamente durante la sincronización del estro (12 días), se aplicó una dosis de $250 \mathrm{mg}$ de cloprostenol y otra de 300 UI de eCG (día 10). La inseminación fue por laparoscopía, 52 h después de retirar las esponjas. Se evaluó la respuesta al estro, inicio del estro, tasa de gestación y de parición, prolificidad, concentración de glucosa en sangre, cantidad y tamaño de folículos ováricos. El suplemento con $P C$ mostró efecto $(P<0.05)$, se obtuvieron 0.64 más cabritos al parto. La concentración de glucosa en sangre se incrementó $22.9 \%$. Durante el estro, aumentó $15.3 \%$ la cantidad de folículos ováricos grandes y el diámetro folicular ovárico fue mayor $0.46 \mathrm{~mm}$. La suplementación con propionato de calcio en cabras durante la sincronización del estro con progestágenos incrementa la concentración de glucosa en sangre, la cantidad de folículos ováricos grandes y la prolificidad.

Palabras clave: Caprinos, fertilidad, folículos ováricos, gluconeogénicos, reproducción.

ABSTRACT. Live weight (LW) and energy intake are known to influence fertility in small ruminants and the benefits of supplying calcium propionate (CP) as a blood glucose precursor. The objective was to determine the physiological and reproductive response of goats synchronized with progestogens (intravaginal sponges impregnated with chronolone, $20 \mathrm{mg}$ ) and a CP supplement. The treatments were randomly assigned to 25 goats of $36.2 \pm 8.7$ months and $51.36 \pm 0.47 \mathrm{~kg}$ of LW: T1 Without propionate $(n=13) 60 \mathrm{~mL}$ of water without CP, and T2 With propionate ( $\mathrm{n}=12) 20 \mathrm{~g}$ of diluted $\mathrm{CP}$ in $60 \mathrm{~mL}$ of water. The CP was administered orally daily (10:00-11:00 am) during the synchronization of estrus (12 d), a dose of $250 \mathrm{mg}$ of cloprostenol and another of $300 \mathrm{IU}$ of eCG was applied (day 10). Insemination was by laparoscopy, $52 \mathrm{~h}$ after removing the sponges. The response to estrus, onset of estrus, gestation and delivery rate, prolificacy, blood glucose concentration, number and size of ovarian follicles were evaluated. The CP supplement showed an effect $(P<0.05), 0.64$ more kids were obtained at delivery. Blood glucose concentration increased $22.9 \%$. During estrus, the number of large ovarian follicles increased $15.3 \%$ and the ovarian follicular diameter was greater by $0.46 \mathrm{~mm}$. Calcium propionate supplementation in goats during estrous synchronization with progestogens increases blood glucose concentration, the number of large ovarian follicles, and prolificacy.

Key words: Goats, fertility, ovarian follicles, gluconeogenic, reproduction. 


\section{INTRODUCCIÓN}

Se sabe que el peso vivo y la ingesta energética inciden en la fertilidad de los pequeños rumiantes, aquellos en mal estado nutricional, o que pierden peso, tienen bajo rendimiento reproductivo, condición asociada con pérdidas económicas debido a la baja fertilidad. Los efectos de la nutrición en la función reproductiva están mediados por componentes dietéticos como lípidos, glucosa, aminoácidos y minerales, los cuales tienen una influencia en el eje hipotalámico-adenohipofisario y repercuten directamente en las gónadas (Senosy et al. 2016, Dupont et al. 2016). La variación en los aportes nutricionales de la dieta produce cambios importantes en la tasa de ovulación, en tal caso, estos cambios pueden estar influenciados por el aporte de nutrientes a corto plazo, mucho antes de observar cambios en la condición corporal (Ying et al. 2011, Senosy et al. 2013, Dupont et al. 2014). Estos efectos nutricionales también pueden estar mediados por cambios en la secreción de hormonas metabólicas como la insulina, el factor de crecimiento similar a la insulina tipo 1 (IGF-1), la hormona del crecimiento, a grelina, las hormonas tiroideas y las hormonas producidas y secretadas por el tejido adiposo (leptina, Dupont et al. 2016); sin embargo, existen evidencias de la importancia de la glucosa en la actividad ovárica (Berlinguer et al. 2012). De igual manera, se ha demostrado que las concentraciones de glucosa son diferentes en el líquido folicular de los folículos en diferentes estadios de desarrollo en el mismo individuo. Así, la concentración de glucosa en el líquido folicular es mayor en los folículos más grandes y en los folículos preovulatorios, lo que sugiere regulación hormonal local en el movimiento de glucosa para su uso por los folículos ováricos. Por lo que, la concentración intrafolicular de glucosa, parece ser modulada por el entorno hormonal del folículo ovárico (Dupont y Scaramuzzi 2016).

Los rumiantes absorben poca glucosa mediante la dieta, debido a que las condiciones anaeróbicas del rumen reducen la mayoría de los carbohidratos y los lípidos a una serie de ácidos grasos volátiles (AGV) que proporcionan más de $70 \%$ del suministro de energía. Al respecto, se sabe que, en los rumiantes, la mayor parte de la glucosa de la dieta se transforma en el rumen y se absorbe como AGV (propiónico, acético y butírico); sin embargo, parte de glucosa puede sobrevivir a las condiciones reductoras del rumen para absorberse en el intestino delgado (Dupont y Scaramuzzi 2016). Aunque la gluconeogénesis no ocurre en todos los mamíferos, los rumiantes son particularmente dependientes de ésta para la generación de glucosa. Así, el propionato es el principal AGV que se produce por la fermentación ruminal de los carbohidratos. Este compuesto, sintetiza entre 45 y $60 \%$ de la glucosa en el hígado a partir del ácido propiónico (Zhang et al. 2020).

En los últimos años, precursores de glucosa, como el propilenglicol y el propionato de calcio, han sido utilizados para corregir problemas metabólicos en ganado lechero, donde las demandas energéticas llegan a superar el aporte energético de la dieta (Mendoza-Martínez et al. 2016) en tal caso, el ácido propiónico, que se hidroliza a partir del propionato de calcio en el rumen, se absorbe por el epitelio ruminal, pasa al hígado a través de la vena porta, donde se sintetiza glucosa (Zhang et al. 2020). Por lo anterior, el objetivo del presente estudio fue determinar la respuesta fisiológica y reproductiva de cabras sincronizadas con progestágenos y un suplemento de propionato de calcio.

\section{MATERIALES Y MÉTODOS}

\section{Localización}

El estudio se realizó durante julio (mes en que las cabras se encuentran en periodo reproductivo) en el módulo de ovinos y caprinos de la Granja Experimental del Departamento de Zootecnia de la Universidad Autónoma Chapingo, Texcoco, Estado de México $\left(19^{\circ} 29 \mathrm{LN}, 98^{\circ} 53 \mathrm{LO}\right.$, a $2250 \mathrm{~m}$ de altitud). El clima de la región es $C(w 0)(w) b(i ') g$, que corresponde a un clima templado subhúmedo con lluvias en verano y época seca en invierno, con precipitación y temperatura media anual de $686 \mathrm{~mm}$ y $15.9{ }^{\circ} \mathrm{C}$, respectivamente; siendo mayo el mes más cálido y enero el mes más frío (García 2004). 


\section{Animales utilizados y manejo}

Se utilizaron 25 cabras (cruzas de las razas Alpina, Saanen y Toggenburg, con condición corporal de $2.0 \pm 0.25$ en la escala 1 a 5 ) de $36.2 \pm 8.7$ meses y $51.36 \pm 0.47 \mathrm{~kg}$ de peso vivo (PV). Previo al estudio, las cabras recibieron un tratamiento profiláctico con antiparasitario (Iverfull ${ }^{\circledR}$, Ivermectina, 0.2 $\mathrm{mg} / \mathrm{kg}$ de PV vía $\mathrm{SC})$, reconstituyente $\left(\mathrm{Mu}-\mathrm{Se}^{\circledR}\right.$, Selenito de sodio, $0.25 \mathrm{mg} / \mathrm{kg}$ de PV vía IM) y vitaminas (Vigantol ${ }^{\circledR}$, A: 6000 Ul / kg de PV, D3: 2000 UI / kg de PV, E1 alfa-tocoferolacetato: $1 \mathrm{mg} / \mathrm{kg}$ de PV). La alimentación se ofreció de 9:00 a 10:00 am y se basó en una mezcla de ensilado de maíz (70\%), alfalfa verde (20\%) y concentrado comercial (10\%).

Para la sincronización de estros se utilizaron esponjas intravaginales impregnadas con $20 \mathrm{mg}$ de chronolona (Chronogest ${ }^{\circledR}$ ) con duración de 12 días; en el día 10 de la sincronización, se aplicaron vía IM 250 mg de Cloprostenol (Celosil ${ }^{\circledR}$ ), y $300 \mathrm{UI}$ de gonadotropina coriónica equina (eCG, Novormon ${ }^{\circledR}$ ), siguiendo el protocolo de manejo reproductivo (Figura 1). Doce horas después del retiro de las esponjas, a intervalos de seis horas durante $48 \mathrm{~h}$, se inició la detección de estros con la ayuda de machos cabríos receladores (desviados quirúrgicamente). En el día 14 del protocolo de manejo reproductivo, se exploraron los ovarios de las cabras mediante un equipo de ultrasonografía Vet 10 (Mindray) con un transductor lineal rectal de $5 \mathrm{MHz}$; con el cual se registró la población folicular presente y la medida de los folículos observados; enseguida, se realizó la inseminación artificial mediante la técnica intrauterina por laparoscopía, de acuerdo con lo descrito por McKelvey et al. (1985). A los 40 días post inseminación, se realizó el diagnóstico de gestación mediante ultrasonografía abdominal con un ultrasonido DRAMINSKI ${ }^{\circledR}, 4$ Vet mini.

Al insertar las esponjas, a la mitad del periodo de sincronización y al momento de su retiro, posterior a la administración del PC, se recolectaron $1.0 \mathrm{~mL}$ de sangre por venopunción en la vena yugular con la finalidad de determinar los niveles de glucosa en sangre, la cual se determinó a temperatura ambiente colocando una gota de sangre en una tira reactiva que se leyó en un glucómetro $\left(\mathrm{ONE}-\mathrm{TOUCH}{ }^{\circledR}\right.$, Jhonson y Jhonson) y se registró el resultado de cada cabra.

\section{Tratamientos experimentales}

Las cabras se asignaron al azar a los tratamientos $(\mathrm{T})$ durante el periodo de sincronización del estro: $T 1(n=13): 60 \mathrm{~mL}$ de agua sin PC, y T2 $(n=12): 20 \mathrm{~g}$ de $P C$ diluido en $60 \mathrm{~mL}$ de agua ofrecido diariamente. El suplemento de PC se administró vía oral diariamente (10:00-11:00 am) durante la sincronización del estro (12 días), mediante una jeringa de plástico de $60 \mathrm{~mL}$.

\section{Variables evaluadas}

Respuesta al estro ( $n$ ): cantidad de hembras que presentaron estro en respuesta a la sincronización con esponjas, expresado en porcentaje. Inicio del estro (h): tiempo transcurrido desde el retiro de la esponja, hasta que la cabra presentó el estro. Tasa de gestación (\%): cantidad de cabras gestantes respecto al total de hembras inseminadas por laparoscopía y que no retornaron al estro; multiplicado por 100. Tasa de parición (\%): cantidad de cabras paridas respecto al total de cabras gestantes, multiplicado por 100 . Prolificidad (n): cantidad de crías nacidas con respecto al total de cabras paridas de cada tratamiento. Concentración de glucosa en sangre: relación de la cantidad de glucosa $(\mathrm{mg})$ por volumen de sangre (dL) evaluado en las cabras de cada tratamiento. Cantidad (n) y diámetro de folículos ováricos medianos (4 a $5 \mathrm{~mm}$ ) y grandes (> 6 mm, Bartlewski et al. 2011): conteo de la población folicular ovárica de las cabras de cada tratamiento y su clasificación por tamaño de acuerdo con su diámetro.

\section{Análisis estadístico}

El diseño experimental utilizado fue completamente al azar, los datos se ordenaron por tratamiento y se analizaron con el paquete estadístico SAS (2012). El inicio del estro se analizó con el método de curvas de sobrevivencia Log-Rank (LIFE TEST), el cual calcula estimaciones no paramétricas de la función de distribución de la probabilidad de supervivencia, se utilizó el método de Bonferroni, el cual analiza diferencias entre medias para la media de 


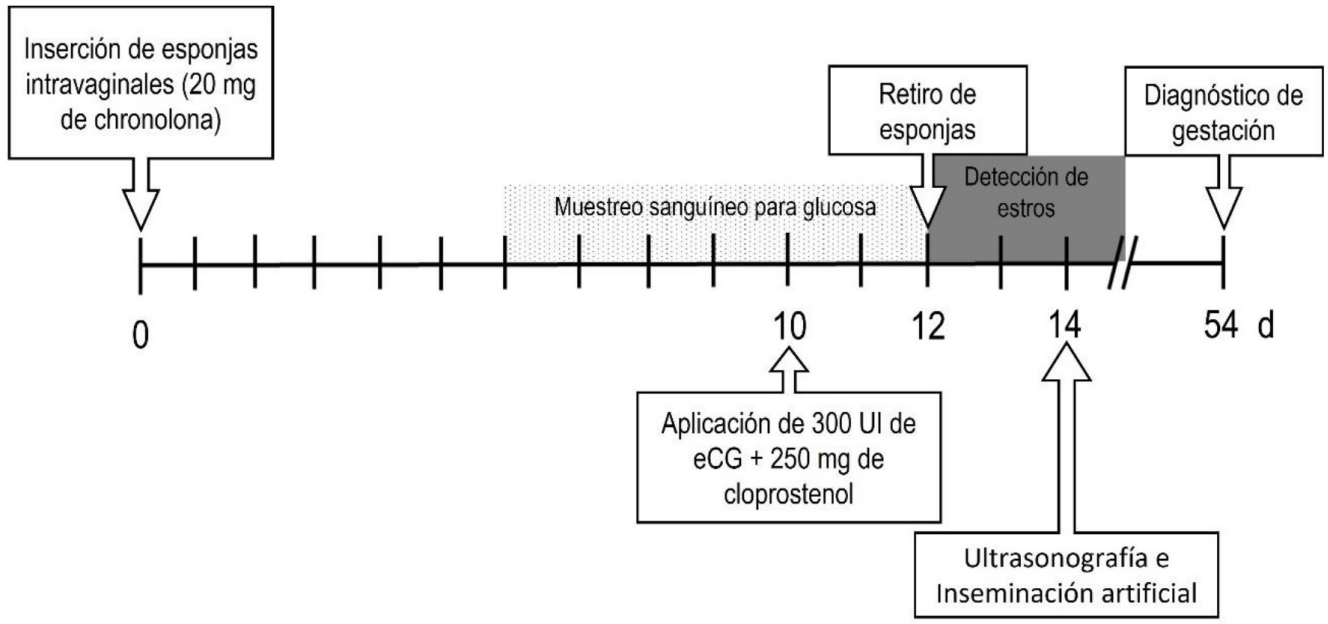

Figura 1. Protocolo de la sincronización del estro en cabras con progestágenos y gonadotropinas.

todos los efectos. Las variables respuesta al estro, tasa de gestación y tasa de parición se analizaron por el procedimiento de regresión logística con el modelo: $P(Y 7 X)=\frac{e^{\beta_{0}+\beta_{1} x}}{1+e^{\beta_{0}+\beta_{1} x}}$ Donde: $\mathrm{P}(\mathrm{Y} / \mathrm{X})$ : Probabilidad de que una cabra presentó estro, se gestó y parió, dado el tratamiento $X(\mathrm{~T} 1$ o T2). e: 2.7. $\beta 0$ y $\beta 1$ : Coeficientes del modelo. Y: Respuesta (si o no). X: Tratamiento. La prolificidad se analizó por el procedimiento de modelos generalizados (PROC GENMOD). Los datos de concentraciones de glucosa en sangre se analizaron por el procedimiento GLM. La cantidad y el tamaño de los folículos ováricos se analizaron por tablas de contingencia, las cuales revelan asociaciones entre dos variables; y los conteos observados, determinan la asociación entre las variables categóricas.

\section{RESULTADOS}

No hubo diferencias $(P>0.05$; Tabla 1) en la respuesta al estro, las cabras suplementadas con PC presentaron $100 \%$ de respuesta al estro en comparación con el tratamiento sin PC, solo el $76 \%$ de las cabras respondió al estro.

La distribución del estro mostró mejor agrupación en el tratamiento sin $P C(P<0.05)$, ya que el $40 \%$ de las cabras presentaron estro a las $20 \mathrm{~h}$ y el $60 \%$ restante, a las $25 \mathrm{~h}$ después de retirado las esponjas intravaginales; mientras que, en las cabras suplementadas con PC, el inicio del estro fue más disperso en el tiempo (Figura 2), pues tan sólo el 33.3\% de las cabras presentaron estro a las $20 \mathrm{~h}$, el $50 \%$ a las $25 \mathrm{~h}$ y el $16.6 \%$ a las $46 \mathrm{~h}$ después de retirar las esponjas intravaginales. La distribución del estro en el tratamiento con $\mathrm{PC}$ se presentó en $26 \mathrm{~h}$, entre las 20 y 46 h $(P<0.05)$; mientras que, en el tratamiento sin PC, el estro se presentó en $5 \mathrm{~h}$, entre las 20 y 25 h.

No hubo efecto de tratamiento $(P>0.05)$ en las tasas de gestación y tasa de parición. Las diferencias de las cabras diagnosticadas gestantes y las que lograron el parto del tratamiento sin PC, mostró una disminución del $7.65 \%$, debido a las pérdidas de gestación ocasionados por factores externos a la investigación. La prolificidad fue diferente $(P<0.05)$ entre tratamientos. El tratamiento con PC aumentó 0.64 cabritos nacidos por cabra (Tabla 2 ).

La concentración de glucosa en sangre se incrementó $(P<0.05)$ en las cabras suplementadas con $P C$, y fue diferente $(P<0.05)$ al final del periodo de sincronización. Se observó $22.9 \%$ más glucosa en sangre en las cabras que recibieron el suplemento con PC con respecto a las del tratamiento sin PC (Figura 3). Antes de retirar las esponjas intravaginales, la cantidad de folículos ováricos fue similar ( $P$ $>0.05)$ entre tratamientos, donde se observó mayor población de folículos de $4 \mathrm{~mm}$ o menor diámetro (67 
Tabla 1. Respuesta al estro e inicio del estro en cabras sincronizadas con esponjas intravaginales, gonadotropinas y tratadas con o sin propionato de calcio.

\begin{tabular}{cccc}
\hline Tratamiento & $\mathrm{n}$ & Respuesta al estro $(\%)$ & Inicio del estro $(\mathrm{h})^{\dagger}$ \\
\hline T1: Sin propionato de calcio & 13 & 76.9 & $23.0 \pm 0.87^{a}$ \\
T2: Con propionato de calcio & 12 & 100 & $26.8 \pm 2.67^{b}$ \\
\hline$a, b$ Valores con distinta literal en columna son diferentes $(\mathrm{P}<0.05) .{ }^{\dagger}$ Media $^{ \pm}$error \\
estándar.
\end{tabular}

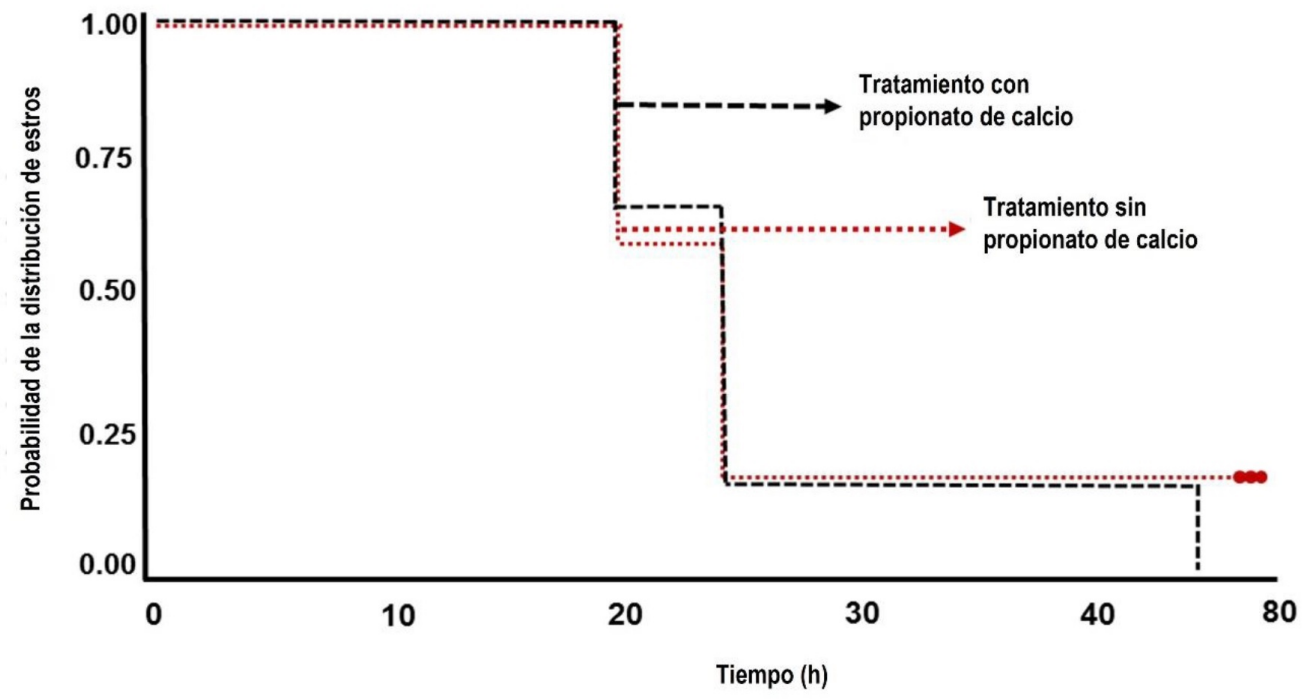

$-\infty$ - Datos censurados: animales que no respondieron al tratamiento

Figura 2. Función de la probabilidad para la distribución del estro de cabras sincronizadas con esponjas intravaginales, gonadotropinas y tratadas con o sin propionato de calcio.

Tabla 2. Tasas de gestación y de parición, y prolificidad en cabras sincronizadas con esponjas intravaginales, gonadotropinas y tratadas con o sin propionato de calcio.

\begin{tabular}{cccc} 
Tratamiento & Gestación (\%) & Parición (\%) & Prolificidad $(\%)^{\dagger}$ \\
\hline T1: Sin propionato de calcio & $53.8^{a}$ & $46.15^{a}$ & $1.16 \pm 0.16^{a}$ \\
T2: Con propionato de calcio & $41.6^{a}$ & $41.66^{a}$ & $1.80 \pm 0.2^{b}$ \\
\hline$a, b$ Valores con distinta literal en columna son diferentes $(\mathrm{P}<0.05) .{ }^{\dagger}$ Media \pm error \\
estándar.
\end{tabular}

y $76 \%$ ) y en menor cantidad (33 y $24 \%$ ), estas proporciones cambiaron después de retirar las esponjas; sin embargo, durante la presentación del estro se observó mayor proporción (15.3\%) de folículos mayores de $4 \mathrm{~mm}$ de diámetro en las cabras suplementadas con PC respecto a las del tratamiento sin PC y redujeron el porcentaje de folículos menores de $4 \mathrm{~mm}$ de diámetro (Figura 4). El diámetro promedio de los folículos ováricos grandes durante el periodo de estro fue diferente $(P<0.05)$ entre tratamientos, las cabras tratadas con $\mathrm{PC}$ presentaron mayor diámetro (7.2\%) de los folículos grandes con respecto a las del tratamiento sin PC (Figura 5).

\section{DISCUSIÓN}

Aunque existen diferentes protocolos para la sincronización o inducción del estro en cabras, la información sobre los protocolos de manejo reproductivo rentables, orientados al caprinocultor y aplicables en campo es escasa. Por lo general, los protocolos de sincronización del estro en cabras consisten en la inserción de un dispositivo con progestágenos durante 12 a 14 días, en el cual se puede incluir o no una dosis de PG y eCG (Manes y Ungerfeld 2015). La respuesta al estro obtenida en el presente estudio es similar a la reportada por Araujo et al. (2013) quienes la evaluaron en cabras con diferentes inter- 


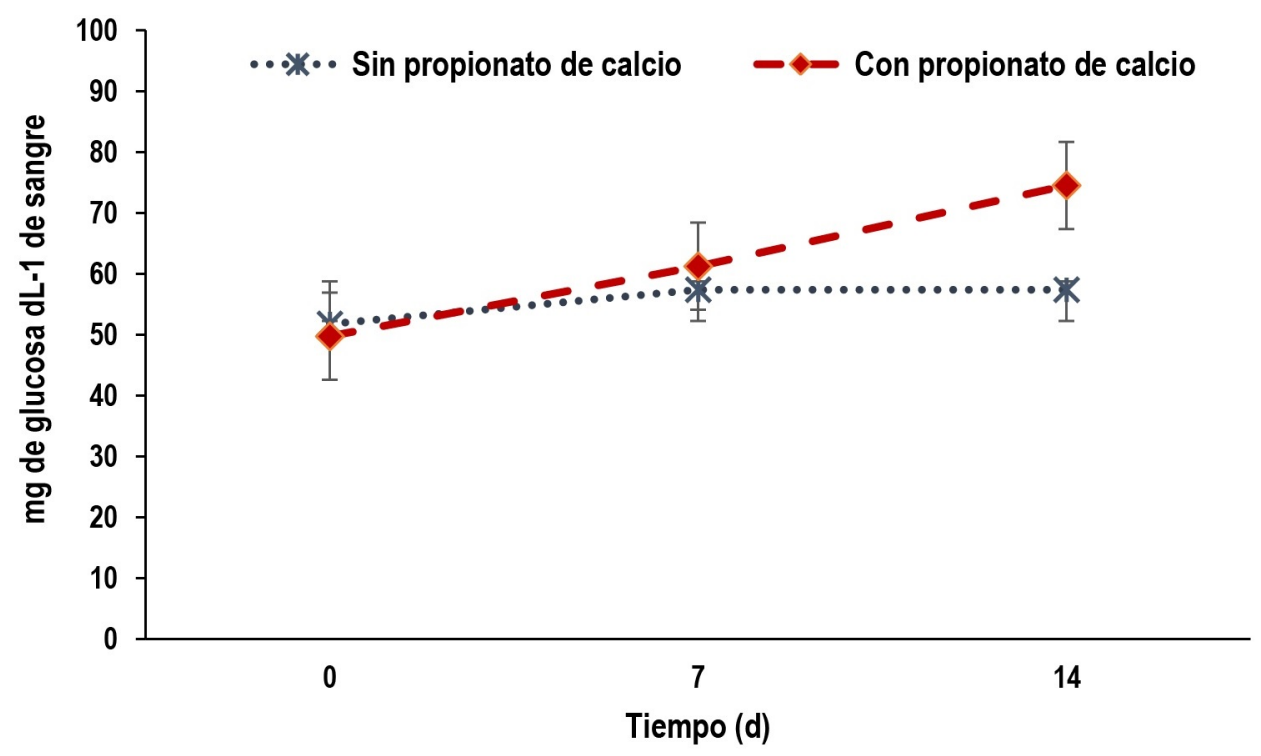

Figura 3. Concentración de glucosa en sangre de cabras sincronizadas con esponjas intravaginales, gonadotropinas y tratadas con y sin propionato de calcio.

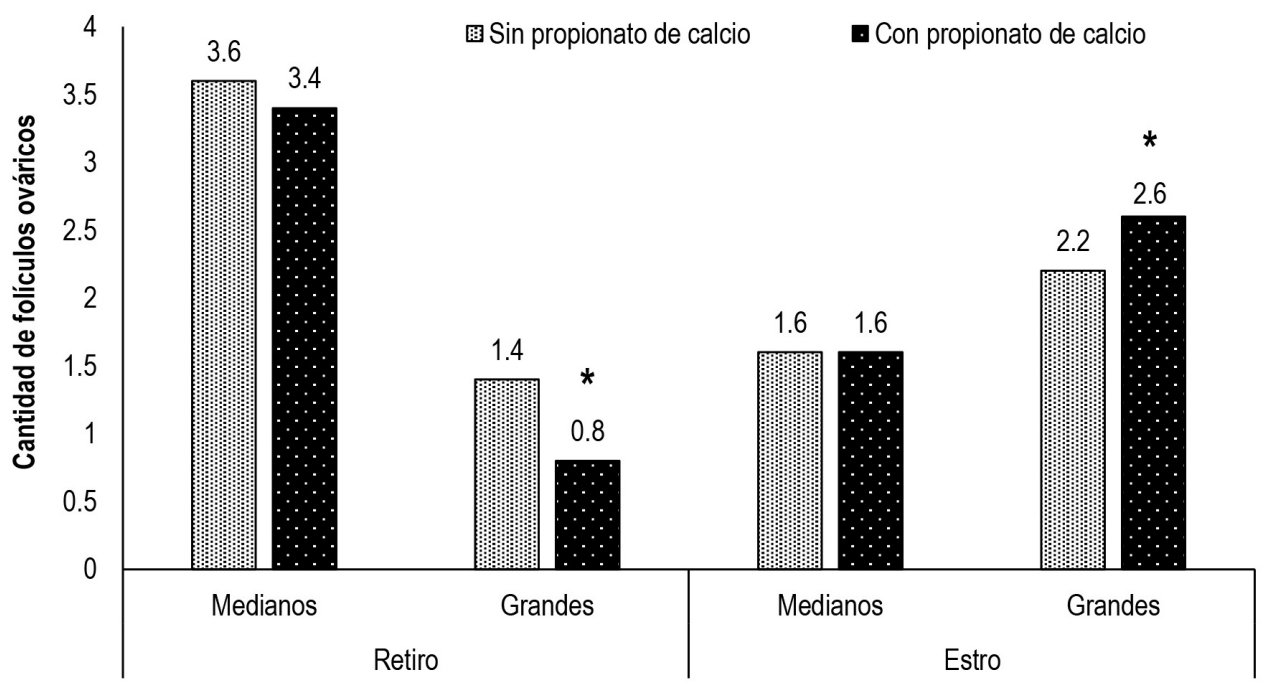

Figura 4. Población folicular ovárica al retiro de la esponja y durante el estro en cabras sincronizadas con esponjas intravaginales, gonadotropinas y tratadas con o sin propionato de calcio ( $\left.{ }^{*}: P<0.05\right)$.

valos de aplicación de esponjas intravaginales. Andrabi et al. (2015) reportaron $100 \%$ de presencia de estros en cabras sincronizadas con doble dosis de cloprostenol con intervalo de nueve días, lo cual difiere con los 10 días del intervalo entre dosis de cloprostenol para obtener el $100 \%$ de respuesta a la sincronización del estro en el presente estudio. Los progestágenos utilizados con mayor frecuencia en la sin- cronización del estro en cabras son la progesterona o los progestágenos sintéticos, los cuales prolongan la fase luteal mientras se mantiene el tratamiento y la acción de la PGF2 $\alpha$ o sus análogos sintéticos, que la acortan inducen la luteólisis (Pérez-Clariget et al. 2012). No obstante, para que el tratamiento con progestágenos sea efectivo, se requiere suficiente gonadotropina necesaria para iniciar los eventos pre- 


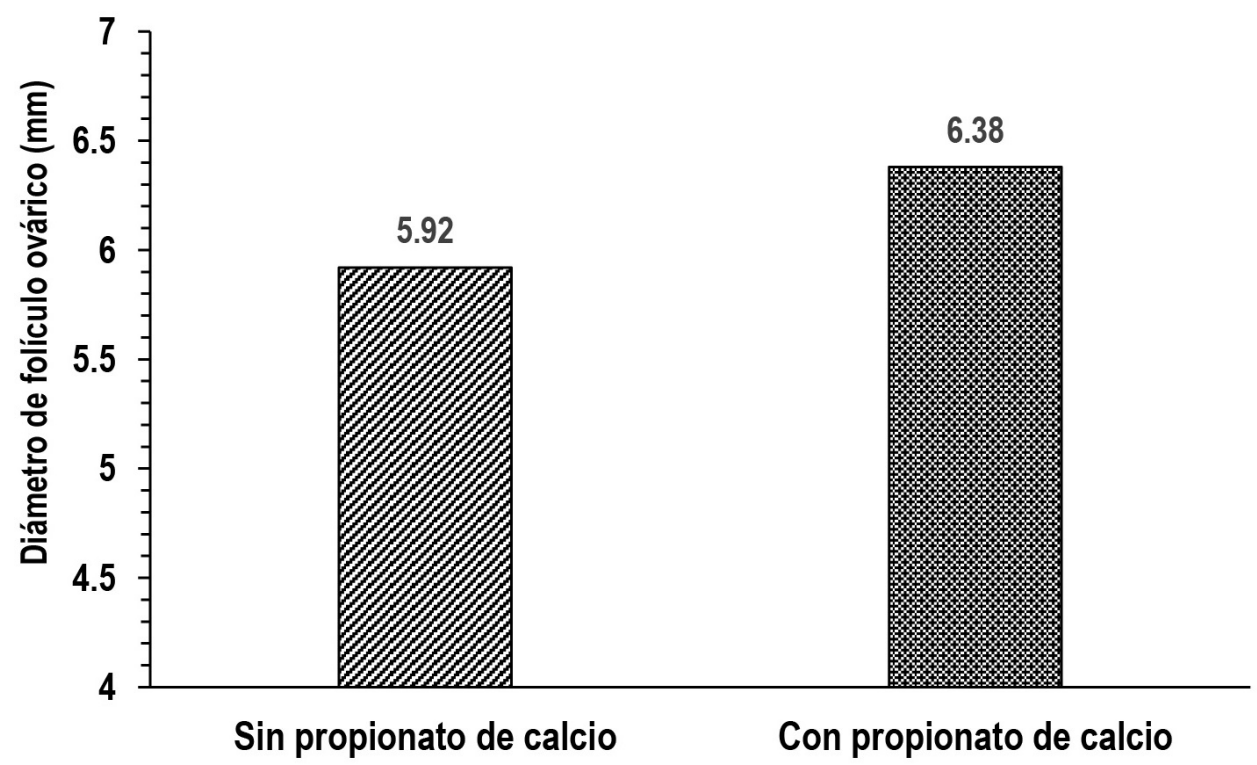

Figura 5. Diámetro promedio de los folículos ováricos grandes durante el estro en cabras sincronizadas con esponjas intravaginales, gonadotropinas y tratadas con o sin propionato de calcio.

ovulatorios, lo cual responde en un aumento en las gonadotropinas endógenas con FSH exógena proveniente de la eCG (Abecia et al. 2011).

Las horas al inicio del estro observadas en el presente estudio son menores a las reportadas por Andrabi et al. (2015) quienes sincronizaron el estro en cabras y obtuvieron $29.6 \pm .60 \mathrm{~h}$ con doble dosis de cloprostenol y $30.2 \pm 8.10 \mathrm{~h}$ con progesterona más cloprostenol. Estos valores son similares a los reportados por Araujo et al. (2013) quienes registraron el inicio del estro entre las 25 y $26 \mathrm{~h}$ posteriores al retiro de las esponjas intravaginales. En protocolos de sincronización del estro, cuando se administra la eCG en combinación con la PGF2a, permite mayor tiempo de actividad, mejor reclutamiento y maduración de folículos y ovocitos (Abecia et al. 2011). No obstante, la presentación del estro varía de 24 a 96 h y depende de edad, raza, temporada, presencia de macho y protocolos de manejo reproductivo (Fatet et al. 2011). En el presente estudio, el inicio del estro en las cabras sincronizadas con cronolona, $P G$ y eCG pudo deberse a la función del desarrollo folicular y la acción del estradiol para la respuesta a la manifestación externa del estro (Abecia et al. 2012).
Las tasas de gestación y de parición son menores que los reportados por Sen y Onder (2016) quienes obtuvieron 82.5 y $80 \%$ de parición en cabras sincronizadas con esponjas y eCG, en contraste con lo reportado por Yotov et al. (2016) quienes indicaron menor fertilidad (33.3 y $45.2 \%$ ) en cabras sincronizadas con FGA y eCG.

La prolificidad obtenida en el presente estudio coincide con lo reportado por Pérez-Clariget et al. (2012) quienes observaron diferencias entre razas en la prolificidad de cabras sincronizadas con esponjas intravaginales, donde las cabras mestizas y criollas mostraron prolificidad de 1.3, mientras que aquellas de razas Nubia y Saanen, prolificidad de 1.8. De igual manera, Sen y Onder (2016) reportaron en cabras sincronizadas con esponjas intravaginales y eCG, prolificidad entre 1.27 y 1.65 , valores similares a los del presente estudio. Existen evidencias de que la suplementación con energía seis días previos a la ovulación incrementa la cantidad de folículos ováricos grandes y, aunque aún no son claros los mecanismos mediante los cuales se promueve el desarrollo folicular ovárico, al parecer están mediados por incrementos en los niveles sanguíneos de glucosa, insulina y estradiol (Habibizad et al. 2015). Al respecto, 
Nogueira et al. (2017) mencionan que los ovarios son sensibles a los estímulos de energía, ya que con la administración de 1.5 veces el requerimiento de energía para mantenimiento durante periodos cortos ( 6 a 9 días) son suficientes para originar la mayor cantidad de folículos ováricos y con ello, incrementar la tasa de ovulación. El uso de gluconeogénicos como el glicerol, se ha utilizado con la finalidad de mejorar la actividad folicular ovárica, con el cual, se ha incrementado el crecimiento folicular ovárico con el consecuente incremento en el porcentaje de parición y la proporción de partos dobles (Bezerra de Oliveira et al. 2016).

Las concentraciones de glucosa en sangre son similares a las reportadas por Zabaleta et al. (2012) quienes determinaron concentraciones en cabras durante el periodo seco y al inicio de lactancia, y registraron 45 a 49 y 45 a $43 \mathrm{mg} \mathrm{dL}^{-1}$, respectivamente; lo cual coincide con los valores iniciales reportados en el presente estudio. Al respecto, Senosy et al. (2016) suplementaron cabras sin producción de leche con 130 veces los valores de mantenimiento y reportaron concentraciones de glucosa entre 90 y $109 \mathrm{mg} \mathrm{dL}^{-1}$, valores superiores a los observados en el presente estudio ( 61.25 y $74.5 \mathrm{mg} \mathrm{dL}^{-1}$, respectivamente); donde las cabras mantenidas con niveles normales de energía, mostraron concentraciones entre 69 y $83 \mathrm{mg} \mathrm{dL}^{-1}$, valores similares a los observados en el presente estudio; sin embargo, estos autores observaron que las concentraciones de glucosa se incrementan a partir del retiro de la esponja hasta alcanzar su valor máximo en el segundo y tercer día post retiro, y después los valores descienden. Lo cual coincide con lo reportado por Scaramuzzi et al. (2015) quienes administraron infusiones de glucosa y observaron que los valores en sangre incrementaron de 55 hasta $115 \mathrm{mg} \mathrm{dL}^{-1}$ en las primeras $24 \mathrm{~h}$, posteriormente decayeron hasta presentar una concentración menor de $50 \mathrm{mg} \mathrm{dL}^{-1}$, valor similar a lo observado en el tratamiento testigo del presente estudio. Alves et al. (2019) suplementaron con diferentes fuentes energéticas a cabras sincronizadas para la recuperación de embriones y observaron que conforme aumentaron los días de suplementación, la proporción de folículos ováricos grandes ( $>4 \mathrm{~mm}$ de diámetro) se incrementó hasta 3.8 folículos por cabra en promedio, lo cual coincide con los resultados obtenidos en el presente estudio. Al respecto, Nogueira et al. (2016) reportaron que la suplementación con diferentes niveles de energía no produjo cambios en la cantidad de folículos ováricos, pero incrementó la tasa de ovulación; sin embargo, al suplementar con maíz (hasta 1.5 veces los valores de mantenimiento) durante la sincronización del estro en cabras, observaron que al retiro de los CIDR, la cantidad de folículos ováricos grandes fue mayor en cabras sin suplementación, y presentaron casi 2.5 folículos grandes en promedio (Nogueira et al. 2017). Mientras que Alves et al. (2019) observaron un incremento en el tamaño de folículos ováricos en cabras sincronizadas y suplementadas con fuentes energéticas con respecto a las que no se suplementaron, y presentaron un tamaño máximo mayor que $6 \mathrm{~mm}$, valores similares a los observados en el presente estudio. En tanto que Nogueira et al. (2017) mencionaron que con la suplementación con maíz (hasta 1.5 veces), los requerimientos de mantenimiento en cabras incrementan el diámetro de folículos ováricos hasta $0.5 \mathrm{~mm}$, tendencia similar a la observada en el presente estudio y Meza-Herrera et al. (2019) indicaron que al suplementar con cladodios de Opuntia megacantha Salm-Dyck enriquecidos, las cabras incrementaron la cantidad y el diámetro de cuerpos lúteos.

El incremento en la prolificidad observada en el presente estudio se relaciona con el aumento en la cantidad de folículos ováricos grandes en el periodo de estro. Hernández-Cerón y GutiérrezAguilar (2013) indicaron que la prolificidad en los pequeños rumiantes está determinada por la cantidad de los folículos ováricos que ovulan, la tasa de fertilización y la sobrevivencia embrionaria. Así, la tasa ovulatoria puede incrementarse por un aumento en la cantidad de folículos ováricos dependientes de gonadotropinas; sin embargo, las gonadotropinas actúan con base en la fase de desarrollo folicular que se encuentren, como lo indican Guo et al. (2019) quienes observaron que la FSH ejerce mayor efecto en los folículos ováricos pequeños y medianos, y que la sensibilidad a la acción de las gonadotropinas 
se incrementa cuando existe una suplementación energética. De igual manera, la $\mathrm{LH}$ ejerce mayor acción en los folículos grandes, donde la sensibilización a esta hormona se incrementó con la suplementación energética de los animales. Lo anterior, es posible debido a la expresión de genes que regulan la síntesis de esteroides en los folículos ováricos; por lo tanto, el incremento en las concentraciones de glucosa en sangre observados en el presente estudio puede estar relacionado con el incremento en folículos ováricos grandes y su sensibilidad a la acción de las gonadotropinas, ya que se ha demostrado la importancia de la glucosa en los folículos ováricos en desarrollo. Dupont y Scaramuzzi (2016) indicaron que la concentración de glucosa en el ovario es necesaria para el estímulo ovulatorio debido a que es el sustrato energético más importante para el ovario. Shabankareh et al. (2013), al evaluar las concentraciones de glucosa en el líquido de folículos ováricos en diferentes estadíos de crecimiento en vacas, observaron que, con el incremento en el tamaño del folículo, existe un aumento en las concentraciones de glucosa en el líquido folicular, y se obtienen mayores concentraciones de glucosa en los folículos ováricos de mayor diámetro.

La suplementación con propionato de calcio es un recurso eficiente para incrementar las concentraciones de glucosa en sangre de rumiantes. AbdelLatif et al. (2016) mencionaron que en búfalas suple- mentadas con propionato de calcio y propilenglicol aumentaron los niveles de glucosa en sangre durante el último tercio de gestación y la primera parte de la lactancia y en específico, el propionato de calcio permitió mayor aumento de las concentraciones de glucosa en sangre, esto debido a que el propionato de calcio en el ambiente ruminal es hidrolizado en $\mathrm{Ca}^{+}$ y ácido propiónico, el cual es absorbido a través del epitelio ruminal, que pasa al hígado por medio de la vena porta, donde se sintetiza glucosa. El propionato es un metabolito anaplerótico obligatorio para el ciclo del ácido tricarboxílico (TCA), entra en el ciclo del TCA mediante succinato, proporciona carbonos que pueden permanecer dentro del ciclo de TCA o extraerse del ciclo de gluconeogénesis (Zhang et al. 2020).

\section{CONCLUSIONES}

La suplementación con propionato de calcio en cabras durante la sincronización del estro con progestágenos incrementa los niveles de glucosa en sangre, aumenta la cantidad y el tamaño de folículos ováricos grandes al final de la sincronización del estro, e incrementa la prolificidad; pero no afecta la fertilidad, la respuesta, el inicio y la distribución del estro.

\section{LITERATURA CITADA}

Abdel-Latif MA, EL-Gohary ES, Gabr AA, El-Hawary AF, Ahmed SA, Ebrahim SA, Fathala MM (2016) Impact of supplementing propylene glycol and calcium propionate to primiparous buffalo cows during the late gestation and early lactation period on reproductive performance and metabolic parameters. Alexandria Journal of Veterinary Sciences 51: 114-121.

Abecia JA, Forcada F, González-Bulnes A (2011) Pharmaceutical control of reproduction in sheep and goats. Veterinary Clinics Food Animal Practice 27: 67-79.

Abecia JA, Forcada F, González-Bulnes A (2012) Hormonal control of reproduction in small ruminants. Animal Reproduction Science 130: 173-179.

Alves JPM, Linhares CC, Rossetto R, Pessoa da Silva C, Oliveira IT, Bertolini M, Rondina D (2019) Impact of short nutrient stimuli with different energy source on follicle dynamics and quality of oocyte from hormonally stimulated goats. Reproduction Domestic Animals 54: 1206-1216.

Andrabi SMH, Anwar H, Mehmood A (2015) Efficacy of short-term estrus synchronization protocols and timed 
artificial insemination in subtropical goats. Short communication. The Journal of Animal \& Plant Sciences 25: 298-300.

Araujo PAC, Zandonadi F, Gonçalves de Souza JM, Ferreira da Fonseca J (2013) Short, medium or long-term hormonal treatments for induction of synchronized estrus and ovulation in Saanen goats during the nonbreeding season. Revista Brasileira de Zootecnia 42: 168-173.

Bartlewski MP, Baby TE, Giffin JL (2011) Reproductive cycles in sheep. Animal Reproduction Science 124: 259-268.

Berlinguer F, Gonzalez-Bulnes A, Contreras-Solís I, Spezzigu A, Torres-Rovira L, Succu S, Naitana S, Leoni G (2012) Glucogenic supply increases oocyte developmental competence in sheep. Reproduction, Fertility and Development 24: 1055-1062.

Bezerra-de-Oliveira FB, Pessoa-da-Silva C, Linhares-Fernandes CC, de-Sousa FC, Silva AM, Nunes-Pinheiro DC, de-Souza-Rodrigues LF, Rondina D (2016) Ovarian follicular growth in Morada Nova sheep supplemented with crude glycerin before mating. Revista Acadêmica Ciência Animal 14: 235-244.

Dupont J, Scaramuzzi RJ (2016) Insulin signaling and glucose transport in the ovary and ovarian function during the ovarian cycle. Review article. Biochemistry Journal 473: 1483-1501.

Dupont J, Scaramuzzi RJ, Reverchon M (2014) The effect of nutrition and metabolic status on the development of follicles, oocytes and embryos in ruminants. Animal 8: 1031-1044.

Dupont J, Scaramuzzi RJ, Froment P (2016) Nutrition et métabolisme: quel lien avec le développement folliculaire et embryonnaire chez les mammifères? INRA Productions Animales 29: 103-116.

Fatet A, Pellicer-Rubio MT, Leboeuf B (2011) Reproductive cycle of goats. Animal Reproduction Science 124: 211-219.

García E (2004) Modificaciones al sistema de clasificación climática de Köppen. $5^{a}$ edición Editorial. Corregida y Aumentada. Instituto de Geografía. Universidad Nacional Autónoma de México. México. 90p.

Guo C, Liu G, Zhao D, Mi Y, Zhang C, Li J (2019) Interaction of follicle-stimulating hormone and stem cell factor to promote primordial follicle assembly in the chicken. Frontiers in Endocrinology 10: 1-13.

Habibizad J, Riasi A, Kohram H, Reza RH (2015) Effect of long-term or short-term supplementation of high energy orhigh energy-protein diets on ovarian follicles and blood metabolitesand hormones in ewes. Small Ruminant Research 132: 37-43.

Hernández-Cerón J, Gutiérrez-Aguilar CG (2013) La somatotropina bovina recombinante y la reproducción en bovinos, ovinos y caprinos. Agrociencia 47: 35-45.

McKelvey WA, Robinson JJ, Aitken RP, Henderson G (1985) The evaluation of a laparoscopic insemination technique in ewes. Theriogenology 24: 519-535.

Manes J, Ungerfeld R (2015) Sincronización de celos en ovejas y cabras con dispositivos intravaginales liberadores de progestágenos: alteraciones en ambiente vaginal y su relación con la fertilidad. Revista Brasileira de Reprodução Animal 39: 104-108.

Mendoza-Martinez GD, Pinos-Rodríguez JM, Lee-Rangel HA, Hernández-García PA, Rojo-Rubio R, Relling AE (2016) Effects of dietary calcium propionate on growth performance and carcass characteristics of finishing lambs. Animal Production Science 56: 1194-1198.

Meza-Herrera CA, Santamaría-Estrada CE, Flores-Hernández A, Cano-Villegas O, García de la Peña C, MacíasCruz U, Calderón-Leyva G, Ángel-García O, Mellado M, Carrillo-Moreno D, Véliz-Derase FG (2019) The 
Opuntia effect upon the out-of-season embryo implantation rate in goats: Corpus luteal number, corpus luteal diameter and serum progesterone. Livestock Science 228: 201-206.

Nogueira DM, Eshtaeba A, Cavalieri J, Fitzpatrick LA, Gummow B, Blache D, Parker AJ (2017) Short-term supplementation with maize increases ovulation rate in goats when dietary metabolizable energy provides requirements for both maintenance and 1.5 times maintenance. Theriogenology 89: 97-105.

Nogueira DM, Cavalieri J, Fitzpatrick LA, Gummowb B, Blache D, Parker AJ (2016) Effect of hormonal synchronization and/or short-term supplementation with maize on follicular dynamics and hormone profiles in goats during the non-breeding season. Animal Reproduction Science 171: 87-97.

Pérez-Clariget R, Garese-Raffo JA, Fleischmann-Techera R, Ganzábal Planinich A, González-Stagnaro C (2012) Sincronización de celos en cabras en estación reproductiva: Uso de esponjas de medroxiprogesterona o aplicación de prostaglandina después de cinco días de detección de celos. Revista Científica 22: 245-251.

SAS (2012) Statistical Analysis Software SAS/STAT ${ }^{\circledR}$. version 9.0.2, Cary, N.C., USA: SAS Institute Inc. http://www.sas.com/en_us/software/analytics/stat.html\#. Fecha de consulta: 28 de enero de 2021.

Scaramuzzi R, Zouaïdi N, Menassol JB, Dupont J (2015) The effects of intravenous, glucose versus saline on ovarian follicles and their levels of some mediators of insulin signaling. Reproductive Biology and Endocrinology 13: 1-14.

Sen U, Onder H (2016) The effect of estrus synchronization programmes on parturition time and some reproductive characteristics of Saanen goats. Journal of Applied Animal Research 44: 376-379.

Senosy W, Mahmoud GB, Abdel-Raheem ShM (2016) Influence of short-term energy supplementation on estrus, ovarian activity and blood biochemistry in Ossimi ewes synchronized with fluorogestone acetate in the subtropics. Theriogenology 88: 152-157.

Senosy W, Abdel-Raheem ShM, Abd-Allah M, Fahmy S, Hassan EH, Derar RI (2013) Effect of transient highenergy diets just after ovulation on ovarian performance and metabolic status in cyclic ewes. Small Ruminant Research 109: 152-155.

Shabankareh HK, Moradi KN, Hajarian H (2013) The influence of the corpus luteum on metabolites composition of follicular fluid from different sized follicles and their relationship to serum concentrations in dairy cows. Animal Reproduction Science 140: 109-114.

Ying S, Wang Z, Wang C, Nie H, He D, Jia R, Wu Y, Wan Y, Zhou Z, Yan Y, Zhang Y, Wang F (2011) Effect of different levels of short-term feed intake on folliculogenesis and follicular fluid and plasma concentrations of lactate dehydrogenase, glucose, and hormones in Hu sheep during the luteal phase. Reproduction 142: 699-710.

Yotov SA, Velislavova DV, Dimova LR (2016) Pregnancy rate in Bulgarian White milk goats with natural and synchronized estrus after artificial insemination by frozen semen during breeding season. Asian Pacific Journal of Reproduction 5: 144-147.

Zabaleta J, Pérez ML, Riera M, Nieves L, Vila V (2012) Concentración de glucosa y triglicéridos en el suero sanguíneo de cabras de la raza canaria durante el período de transición. Revista Científica, FCV-LUZ 22: 225-230.

Zhang F, Nan X, Wang H, Guo Y, Xiong B (2020) Research on the applications of calcium propionate in dairy cows: A Review. Animals 10: 1336. DOI: 10.3390/ani10081336. 